QUASI-INVARIANT MANIFOLDS, STABILITY, AND GENERALIZED HOPF BIFURCATION

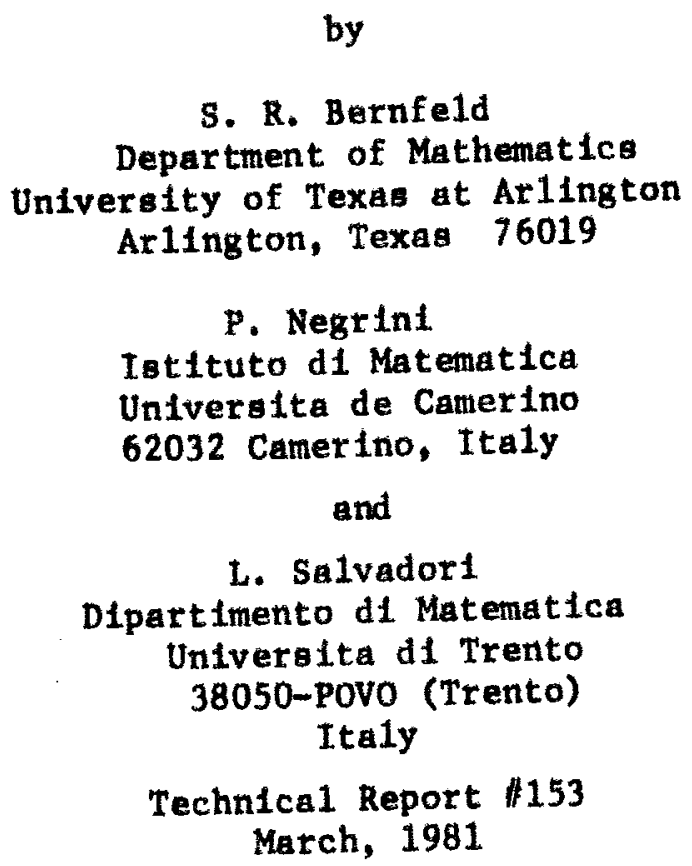




\section{QUASI-INVARIANT MANIFOLDS, STABILITY, AND GENERALIZED HOPF BIFURCATION}

by

S. R. Bernfe1d*, P. Negrint**, and L. Salvador1*

\section{INTRODUCTION}

We are Interested in obtaining an analygls of the bifurcating perfodic orbits arlaing in the generallzed Hopf bifurcation problems in $\mathrm{R}^{\mathrm{n}}$. The existence of theee periodic orbits has often been obtained by uaing such techniqueg as the Lyapunov-Schaidt method or topologlcal degree argumenta (aee Margden and McCracken [8] and Hale [6] and their references). Our approach, on the othar hand, is based upon stability properties of the equilibritm point of the unpetturbed system. Andronov et. al. [1] showed the ftultItilness of this aproach in atudying bifurcation problems in $\mathrm{R}^{2}$ (for more iscent papere gee Negrint and Salvadort [9] and Bernfeld and Salvador1 [2]). in the case of $R^{2}$, In contrast to that of $R^{n}, n>2$, the stability arguments can be effectively applied because of the Poincare-Bendixson theory. B1furcaLon probletie in $\mathrm{R}^{\mathrm{n}}$ can be reduced to that of $\mathrm{R}^{2}$ when two dimenstonal invariant manifolda are known to extst. The existance of such mantfolds occurs, for ande then the unperturbed system contalns only two purely imaginary etgervaluze.

In the papex whe whe be concerned with the general situation in $R^{n}$ th whin the unperturbed system may have several pairs of purely imagtnary

*Thts reseatch was partially gupported by U. S. Army Research Grant DAAG29-80-C-0060.

* Work performed under the ausplces of Italian Counc1l of Research (CNR). 
elgenvalues. To be more precise, let us consider the differential system

$$
\dot{p}=t_{0}(p),
$$

where $f_{0} \in C^{\infty}\left[B^{n}\left(a_{0}\right), R^{n}\right], f_{0}(0)=0$, and $B^{n}\left(a_{0}\right)=\left\{p \in R^{n}:\|p\|<a_{0}\right\}$. Assume the Jacobian matrix $f_{0}^{\prime}(0)$ has two purely lmaginary elgenvalues \pm 1 and that the remalning elgenvalues $\left\{\lambda_{f}\right\}_{j-1}^{n-2}$ satisfy $\lambda_{f} \neq m 1, m=0$ $\pm 1+\ldots$

For those $f \in C^{\infty}\left[B^{n}\left(a_{0}\right), R^{n}\right], f(0)=0$, which are close to $f_{0}$ (In an appropriate topology) consider the perturbed aystem

$$
\dot{p}=f(p) .
$$

We are Interested in determining the number of nontrivia1 periodic orbits of (1.2) lying near the ortgin and having perlod close to $2 \pi$ for those $f$ close to ${ }^{f} 0^{\circ}$

In approaching this problem, we w111 consider for any positive integer k the following property:

(a) (1) there exists a netghborhood $N^{*}$ of $f_{0}$ an $a_{1}>0$ and a number $\delta_{1}>0$ such that for every $f \in N *$ there are at most $k$ nontrivial periodic orbits of $(1.2)$ lying in $B^{n}\left(a_{1}\right)$ whose pertod is in $\left[2 \pi-\delta_{1}, 2 \pi+\delta_{1}\right]$;

(11) for each integer $1,0 \leq f \leq k$, for each $a_{2} \in\left(0, a_{1}\right)$ for each $\delta_{2} \in\left(0, \delta_{1}\right)$ and for each neighborhood $N$ of $E_{0}, N \subseteq N^{*}$, there exists $f \in N$ such that (1.2) has exactly $f$ nontrivial periodic orbits lying In $B^{n}\left(a_{2}\right)$ whose period to in $\left[2 \pi-\delta_{2}, 2 \pi+\delta_{2}\right]$;

(111) for any $T \in\left(0, a_{1}\right), \bar{\delta} \in\left(0, \delta_{1}\right)$ there exists a nelghborhood $\bar{N}$ of $f_{0}, \vec{N} \subseteq N^{*}$ such that if $f \in \vec{N}$ and if $\gamma$ is a periodic orbit of 
(1.2) 1ying in $\mathrm{B}^{\mathrm{n}}\left(\mathrm{a}_{1}\right)$ whose period is in $\left[2 \pi-\delta_{1}, 2 \pi+\delta_{1}\right]$ then $\gamma$ 11ee in $B^{n}(\vec{B})$ with period in $[2 \pi-\vec{\delta}, 2 \pi+\bar{b}]$.

In contrast to (a) another property which we constder in this paper 18:

(A) For any nelghborhood $\mathrm{N}$ of $\mathrm{f}_{0}$, for any integer $1 \geq 0$, for any $\in\left(0, a_{1}\right]$, and for any $\delta>0$ there exlst $\in \ddot{E} N$ such that (1.2) has $f$ nontrivial pertodic orbits lying in $\mathrm{g}^{\mathrm{n}}(\mathrm{a})$ whose period is in $\left[2 \pi-\delta_{2}, 2 \pi+\delta_{2}\right]$.

In $R^{2}$. Andronov et.a1. [1] proved that property $(a)((1),(11))$ is a consequence of the ortgin of $(1.1)$ being h-asymptotically stable or $h$ completely ungtable where $h$ is an odd integer and $k=\frac{h-1}{2}$. The origin of (1.1) In $R^{n}$ is sald to be h-asymptotically stable (h-completely unstable) If $h$ lo the smallest positive integer such that the origin of (1.2) is asymptotically stable (completely unstable) for all f for which $f(p)-f_{0}(p)=o\left(\|p\|^{h}\right)$; that is $h$ ts the mallest positive integer such that asymptotic stability (complete ingtability) of the or $1 g \ln$ for (1.1) 1s recognizable by Inspecting the terma up to order $h$ in the Taylor expansion of $f_{0}$ (aee Negrint and Salvadort [9] for further Information on $h$ asymptotic otability). In a recent paper Bernfeld and $5 a 1$ vadori $[2]$ In $R^{2}$ extended the results of Andronov et.al. [1] by proving property (a) 1 a equivalent to the $h$-asymptotic stability (h-complete instability) of the orlgin of (1.1) (where again $k=\frac{h-1}{2}$ ). It wag also shown that property (A) Is equivalent to the case in which the or $1 \mathrm{~g}$ in of (1.1) is nether h-asymptoti cally stable nor h-completely unstable for any positive integer $h$. The problem in $\mathrm{R}^{\mathrm{n}}$ was first considered by Chafee [5]. Uaing the Lyapunov-Schnidt method he obtained a determining equation $\psi(\xi, f)=0$ 
where $\psi$ is a measure of the amplitude of the bifurcating period orbits of (1.2) and $f$ represents the right hand side of (1.2). By aseuming that the multiplicity of the zero root of $\psi\left(\cdot, f_{0}\right)$ la finite number $k$, he proved that property (a) holds for this $k$.

Our goal in this paper 18 to relate the number $k$ in property (a) wth the conditional asymptotic stablifty properties of the origin for a differential system which is close in some sense to the unperturbed system (1.1). These stability properties are preclsely the h-asymptotic stability (h-complete ingtabl11ty) of the orfgen for a particular differential equation $\left(s_{h}\right)$ in $R^{2}$. The conetruction of $\left(s_{h}\right)$ as well as the recognttion of the h-asymptotic stability (h-complete Instablilty) of the or $1 g$ in of $\left(s_{h}\right)$ can be accomplished by solving IInear algebraic systems. Indeed, these stablifty propertieg can be recognized by applylng the classical Poincare procedure (see [9] of [10]). Thus, the number $k, k=\frac{h-1}{2}$, can be determined using elementary algebralc techniques. The analysis of our problem is completed by observing that when the origin for $\left(s_{h}\right)$ is netther $h-$ asymptotically stable nor h-completely unstable for every $h>0$ then property (A) holds.

The main Ingredients of our analysis are: (1) the construction of a quasi-invartant mantfold $\sum_{h}$ for the unpertubed system (1.1): (11) the use of the Polncare map along a particular set of solutions of (1.1) which are intelaliy close to $L_{h}$.

In conclusion, the quantitative problem of determining the number of blfurcating perlodic solutions of the perturbed system $(1.2)$ can be reduced to an analysis of the qualltative behavior of the flow near the origin of a two dimensfonal system appropriately related to the unperturbed aystem 
(1.1). In addition, an algebrate procedure allows for a concrete solution to the problem.

Fina11y, we remark that an announcament of our results was presented at a conference in Trento, Italy [3].

\section{RESULTS}

We w111 endow the epace $C^{\infty}\left[b^{n}\left(a_{0}\right), R^{n}\right]$ with the following topology: define a function $\|\mid \cdot\|$ mapping $C^{\infty}\left[B^{n}\left(a_{0}\right), n^{n}\right]$ into $R$ as

$$
\|f\|=\sum_{l=0}^{\infty} \frac{\|f\|^{(\ell)}}{2^{\ell}\left(1+\|+\|^{(l)}\right)}
$$

where $\|f\|^{(l)}$ denotes the usual $c^{(l)}$-suptemum norm of $f$ on $B^{n}\left(a_{0}\right)$. Then $C^{\infty}\left[B^{n}\left(a_{0}\right), R^{n}\right]$ is a metric 1inear gpace under $\| I \cdot|l|$. For any vector $w \in R^{n}$ we sha11 denote by $\|$ w the Euclidean norm of $w$.

By an approprtate change of coordinateg depending on $f$ we may write systems (1.1) and $(1.2)$ respectively in the form

$$
\begin{aligned}
& \dot{x}=-y+X_{0}(x, y, z) \\
& \dot{y}=x+Y_{0}(x, y, z) \\
& \dot{z}=A_{0} z+z_{0}(x, y, z)
\end{aligned}
$$

and

$$
\begin{aligned}
& \dot{x}=\alpha x=B y+X(x, y, z, f) \\
& \dot{y}=a y+B x+Y(x, y, z, F) \\
& \dot{z}=A z+z(x, y, z, f) .
\end{aligned}
$$

For each fixed $f, \alpha$ and $B$ are constants satfifying $\alpha\left(f_{0}\right)=0, B\left(f_{0}\right)=1$ 
and $A$ Ls an $(n-2) \times(n-2)$ constant natrlx satisfying $A\left(f_{0}\right)=A_{0}$. Moreover, for flued $\mathrm{F}, \mathrm{X}$ and $\mathrm{Y}$ belong to $\mathrm{C}^{\infty}\left[\mathrm{B}^{\mathrm{n}}\left(\mathrm{a}_{0}\right), \mathrm{f}\right], z$ belongs to $C^{\infty}\left[b^{n}\left(a_{0}\right), k^{n-2}\right]$, and $X, Y, Z$ are of order greater than one. The elgenvalues of $A_{0},\left\{\lambda_{j}\right\}_{j=1}^{n-2}$ satisfy the condition that $\lambda_{j} \neq$ min $n$ m, $01 \ldots$. We now constder an $(n-2)$ dimensional polynomial of sone degree $h$, $\mathrm{h} \geq 1$, given by

$$
\phi^{(h)}(x, y)=\phi_{1}(x, y)+\ldots+\phi_{h}(x, y)
$$

where $\phi_{f}(x, y)$ ls homogeneous of degree $j$. We attempt to determine $\phi_{1}, \ldots, \phi_{h}$ in order to oftain along the solutions of $(2,1)$

$$
\left.\left[\frac{d}{d t}(z-\phi)(h)(x, y)\right)\right]_{z=\phi}(h) \underset{(x, y)}{o\left(x^{2}+y^{2}\right)^{h / 2}}
$$

that 1s, we have to satiafy

$$
\begin{aligned}
& \frac{\partial \phi^{(h)}(x, y)}{\partial x}\left[-y+x_{0}(x, y, \phi(h)(x, y))\right] \\
& +\frac{\partial \phi^{(h)}(x, y)}{\partial y}\left[x+y_{0}\left(x, y, \phi^{(h)}(x, y)\right)\right] \\
& =A_{0} \phi^{(h)}(x, y)+z_{0}\left(x, y, \phi^{(h)}(x, y)\right)+o\left(x^{2}+y^{2}\right)^{h / 2} .
\end{aligned}
$$

Thig Implies for every $f \in[1, \ldots, n\}, \phi_{j}$ has to satify the partial differential equation

$$
\frac{\partial \phi}{\partial y} x-\frac{\partial \phi_{j}}{\partial x} y=A_{0} \phi_{j}+U_{j}
$$

where $U_{j}$ is ar $(n-2)$ dimensional homogeneous polynomial of degree $I$ depending on the functions ${ }^{\frac{1}{7}} 1 \ldots \phi_{j-1}$. Under the assumptions on $A_{0}$ $(2.6)$ has andque solution and can be solved recutelvely by observing that $\phi_{1}(x, y) \equiv 0$ (ace for Instance Bibikov $\left.[4]\right)$. 
The two dimensional surface $z \phi^{(h)}(x, y)$ is tangent the ortgin to the elgenspace correaponding to the efgenvalues 1 . Thig gurface w11 be called a quas-1nvariant mantold of order h.

Given any $h>0$ define the following two dinenglonal system

$\left(S_{h}\right)$

$$
\begin{aligned}
& \dot{x}=-y+x_{0}\left(x, y, \phi{ }^{(h)}(x, y)\right) \\
& y=x+y_{0}\left(x, y, \phi{ }^{(h)}(x, y)\right) .
\end{aligned}
$$

(Th1s is the aystem referred to in the introduction).

We distingutsh the two posatble cases:

I. There exlats $h>1$ (and then h must be odd) such that $x \equiv y \equiv 0$

18 elther h-asymptotically gtable or h-completely unstable for $\left(\mathrm{S}_{\mathrm{h}}\right)$.

II. Case $\mathrm{I}$ does not hold.

We are now able to gtate out main result.

Theorem 1. In Case I property (a) holds with $k=\frac{h-1}{2}$. In Case II, property (A) holds.

If a11 the elgenvalues of $A_{0}$ have real part not equal to zero, then for every $h>1$ there extes a $c^{h+1}$ two dinenglonal center manifold

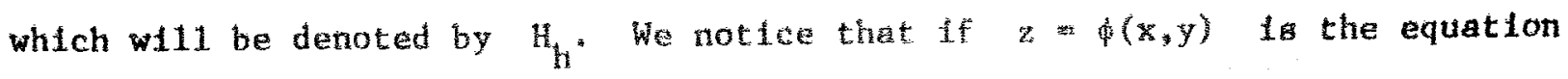
of this center manifold, we can wry to

$$
\phi(x, y)=\phi^{(h)}(x, y)+o\left(x^{2}+y^{2}\right)^{h / 2}
$$

As a consequence of Theorem 1 the following restit holds

Corollary 1. Suppoge that all the elgervalues of $\mathrm{A}_{0}$ have ragl part diffarent than zero. Then: (1) if there extats an h (and $h$ must be odd) such that the origin of the unperturbed aystem (2.1) Is either hasymptoticaliy 
stable or h-completely unstable on $H_{h}$ (that 18 , with respect to initial points on $H_{h}$ ) then $(a)$ holds with $k=\frac{b-1}{2}$; (11) if for every $h>1$ the origin for the unperturbed system (2.1) is netther h-asymptotically stable nor h-completely unstable on $H_{h}$ then (A) holds.

Under some more particular hypotheses on the elgenvalues of $f_{0}(0)$ the stability properties in Theoren 2 can be expressed in terms of the unperturbed system (2.1) whthout any explicit involvement of $H_{h}$. This can be proved by the extension of the polncare procedure $[10] \mathrm{gtven}$ by Lyapunov [7]. Precisely the following reault holds.

Corollary 2. Suppose all the eigenvalues of $A_{0}$ have negative real part. Then (1) if the origin of the unperturbed system (2.1) 18 efther $h$ asymptotically stable or h-ungtable (In the whole) then (a) holds with $k=\frac{h-1}{2} ;$ (11) If for every $h>1$ the or $1 g$ in for the unperturbed syatem (2.1) Is netther h-asymptotically stabie nor h-unstable, then (A) holds. Notice that we are using the concept of h-unstable whose definition 1s analogous to that of h-complete Instability. A similar theorem can be atated when $f_{0}{ }^{\prime}(0)$ has two purely imaginary elgenvalues \pm 1 and the remalning elgenvalueg have positive real part.

\section{PRELIMINARY PROPOSITIONS}

Uaing the transformation

$$
\zeta=z-\phi^{(h)}(x, y)
$$

we can rewrite the unperturbed system (2.1) as 


$$
\begin{aligned}
& \dot{x}=-y+x_{0}^{(h)}(x, y, \zeta) \\
& y=x+y_{0}^{(h)}(x, y, \zeta) \\
& \xi=A_{0} \zeta+W_{0}^{(h)}(x, y, \zeta),
\end{aligned}
$$

where $\left.x_{0}^{(h)}(x, y, 0)=x_{0}\left(x, y, \phi{ }^{(h)}(x, y)\right), y_{0}^{(h)}(x, y, 0)=y_{0}^{(x, y, \phi(h)}(x, y)\right)$. From $(2.4)$ we observe that $\omega_{0}^{(h)}(x, y, 0)$ la of order greater than $h$. Analogously, we can rewtite the perturbed systen $(2.2)$ as

$$
\begin{aligned}
& \dot{x}=\alpha x-B y+z^{(h)}(x, y, \zeta, f) \\
& \dot{y}=\alpha y+B x+y^{(h)}(x, y, \zeta, f) \\
& \dot{\zeta}=A \zeta+w^{(h)}(x, y, \zeta, f),
\end{aligned}
$$

where $X^{(h)}(x, y, 0, f)=X\left(x, y, \phi^{(h)}(x, y), f\right), Y^{(h)}(x, y, 0, f) \neq Y\left(x, y, \phi^{(h)}(x, y), f\right)$ and for fixed $f, X^{(h)}, y^{(h)}, W^{(h)}$ are of order $\geq 2$. Let us aet $I_{\delta}=[2 \pi-\delta, 2 \delta+\delta]$ for any $\delta>0$. We now give the following proposition.

Proposition 1. There exist $E, \delta_{3} L>0$ and a netghborhood $\mathrm{N}_{0}$ of $\mathrm{f}_{0}$ guch that whenever $f \in N_{0}$ and $(x, y, b) \in \gamma$, whete $\gamma$ ls any $T$-periodic orb1t of $(3,2)$ lytng in $B^{n}(\varepsilon)$ with $T \in I_{g^{3}}$ then $\|\zeta\| \leq L\left(x^{2}+y^{2}\right)$.

Proof. Choose $\varepsilon_{1} \in\left(0, a_{0}\right), \delta_{1}>0$ and a nelghborhood $N_{1}$ of $f_{0}$ such that: (1) $\operatorname{det}\left(t-e^{A T}\right) \neq 0$ for $E \in N_{1}$ and $T \in I_{0} ;(11)$ the solutions of (3.2) through the inftal polnt $\left(0, x_{0}, y_{0}, \tau_{0}\right),\left(x\left(t, x_{0}, y_{0}, \zeta_{0}, f\right), y\left(t, x_{0}, y_{0}, \zeta_{0}, f\right)\right.$, $\left.\left(t, x_{0}, y_{0}, \delta_{0}, f\right)\right)$ exist and belong to $B^{n}\left(a_{0}\right)$ for a1l $\left(x_{0}, y_{0}, 5_{0}\right) \in B^{n}\left(\varepsilon_{1}\right)$, $f \in N_{1}$ and $t \in\left[0,2 \pi+\delta_{1}\right]$. Here $I$ is the $(n-2) \times(n-2)$ Identity matrix. Condition (1) can be gatisfied for $t$ cloze to ${ }^{2}$ and $\delta_{1}$ small becauge our assumptions on the elgenvalues of $A_{0}$ implies that $\operatorname{det}\left(\mathrm{I}-\mathrm{e}^{2 \pi \mathrm{A}_{0}}\right) \neq 0$ 
We now want to determine $\left(x_{0}, y_{0}, \xi_{0}\right) \in B^{n}\left(\varepsilon_{1}\right), E \in N_{1}$ and $T \in I_{\delta_{1}}$ which gatisfy the condition

$$
\zeta\left(\mathrm{T}, \mathrm{x}_{0}, \mathrm{y}_{0}, \mathrm{r}_{0}, \mathrm{f}\right)=\xi .
$$

From the third equation in $(3.2)$ It follows that $(3.3)$ is equivalent to the equation $F\left(x_{0}, y_{0}, c_{0}, T, f\right)=0$, where

$$
\begin{aligned}
F\left(x_{0}, y_{0}, \zeta_{0}, T, f\right)= & \left(I-e^{A T}\right) \zeta_{0}-\int_{0}^{T} e^{A(T-s)}\left[W ( h ) \left(x\left(s, x_{0}, y_{0}, \zeta_{0}, f\right), y\left(8, x_{0}, y_{0}, \zeta_{0}, f\right),\right.\right. \\
& \left.\left.\zeta\left(s, x_{0}, y_{0}, \zeta_{0}, f\right), f\right)\right] d s .
\end{aligned}
$$

Since $W^{(h)}$ is of order $\geq 2$ in $\left(x, y, r_{3}\right)$ for each $t$ we have $\mathrm{F}\left(0,0,0,2 \pi, \mathrm{F}_{0}\right)=0$ and let $\mathrm{D}_{\tau_{0}} F\left(0,0,0,2 \pi, \hat{f}_{0}\right)=\operatorname{det}\left(1-e^{2 \pi A_{0}}\right) \neq 0$. Then, by the Impliclt function theorem, there exist $\varepsilon \in\left(0, \varepsilon_{1}\right], \delta \in\left(0, \delta_{1}\right]$, $\mathrm{N}_{0} \subseteq \mathrm{N}_{1}$ and $o \in \mathrm{C}\left[\mathrm{B}^{2}(\varepsilon) \times \mathrm{I}_{\delta} \times \mathrm{N}_{0}, \mathrm{R}^{\mathrm{n}-2}\right], \sigma\left(0,0,2 \pi, \mathrm{F}_{0}\right)=0$ such that (a) For every $\left(\mathrm{x}_{0}, \mathrm{y}_{0}, \zeta_{0}\right) \in \mathrm{B}^{\mathrm{n}}(\varepsilon), \mathrm{T} \in \mathrm{I}_{\delta}$, and $\tilde{E} \in \mathrm{N}_{0}$ (3.3) holda if and only if $\zeta_{0}=o\left(x_{0}, y_{0}, T, f\right)$.

(B) $\left\|\sigma\left(x_{0}, y_{0}, T, f\right)\right\| \leq L\left(x_{0}{ }^{2}+y_{0}{ }^{2}\right)$ for some constant $\mathrm{L}>0$ and for $a 11$ $\left(x_{0}, y_{0}\right) \in B^{2}(\varepsilon), T \in I_{j}, f \in N_{0}$

The function $\sigma: 1 \% c^{\infty}$ in $\left(x_{0}, y_{0}\right)$ and its derivativer are continuous In a11 varlables $x_{0}, y_{0}, T, f$. Tor any $T \in I_{g}$ and $f \in N_{0}$ we have $F(0,0,0, T, f)=0$ and then $\sigma(0,0, T, f)=0$. Moreover, det $D_{C_{0}} F(0,0,0, T, f)$ $\operatorname{det}\left(T-e^{A T}\right) \neq 0$ (becatre of $(i)$ and $D_{x_{0}}(0,0,0, T, f)=D_{y_{0}} \mathbf{T}(0,0,0, T, f)=0$, which lmp11es $\mathrm{D}_{\mathrm{x}_{0}} \sigma(0,0, \mathrm{~T}, \mathrm{f})=\mathrm{D}_{\mathrm{y}_{0}} \sigma(0,0, \mathrm{~T}, \mathrm{f})=0$. In part1cular, conslder any T-pertodic solution of $(3.2)$ lying in $B^{n}(\varepsilon)$, wh $T \in I_{\delta}$ and $f \in N_{0}$ and denote 1 ts orbit by $\gamma$. Since $(3.2)$ is autonomous condition (3.3) 1B satisfled for any polnt $(x, y, \zeta) \in \gamma$. Thus, Proposition 1 immediately follows from $(\alpha),(\beta)$ 
The substitution

(3.4)

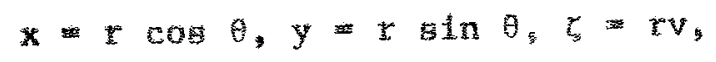

Into (3.2) gives aystem which we write as

$$
\frac{d E}{d \theta}=R^{(h)}(\theta, y, y, f)
$$

$$
\frac{d v}{d 6}=\frac{A}{B} v+n^{(h)}(\theta, r, v, E)
$$

where $R^{(h)}, n^{(h)} E C^{\infty}$. The solutitoni of $(3.5)$ For which $r(\theta) \neq 0$ for all $\theta$ are the orblts of corresponding solutions of $(3.2)$. Moreover, the ortgtn Ia a glution of both $(3.2)$ and $(3.5)$. We denote by $\left(r\left(\theta, c, v_{0}, f\right), v\left(\theta, c, v_{0}, f\right)\right.$ the solution of $(3,5)$ passing through $\left(0, c, v_{0}\right)$. When the solutions $(r(\theta), v(\theta))$ of $(3.5)$ ar known, the corregponding solutions of (3.2) can be completely. determined by solvintro the equation.

$$
\frac{d \theta}{d t}=\theta(0, r(\theta), v(\theta), r)
$$

where $\theta$ ir greater than some postefve number In a nelghborhood of the origin

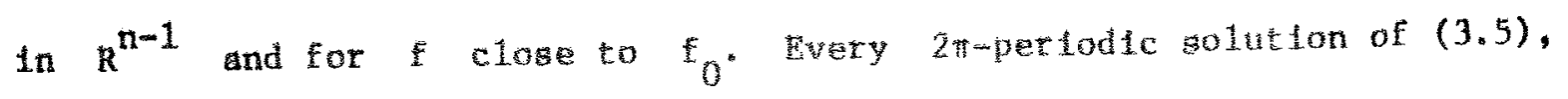
$(r(\theta), v(\theta))$ represent pertodic orble of $(3.2)$ whose period $T$ is given by

$$
T \int_{0}^{2 \pi} \frac{d \theta}{\theta(\theta, t(\theta), v(0), T)}
$$


For any $\varepsilon>0$ denote by $P(\varepsilon)=\left\{(r, v) \in R^{n-1}, r \geq 0,2^{2}+\|v\|^{2}<\varepsilon^{2}\right\}$. We now introduce for system (3.5) property ( $\left.a^{\prime}\right)$ which corresponds to property (a) for system (3.2).

$\left(a^{\prime}\right)(1)$ There exists a nefghborhood $N^{*}$ of $f_{0}$ and an $\varepsilon_{1}>0$ such that for every $f \in N^{*}$ there are at most $k$ nontrivial $2 \pi$-perfodic orbits of (3.5) 1ying in $P\left(E_{1}\right)$.

(11) For each integer $f, 0 \leq f \leq k$, for each neighborhood $N$ of $f_{0}$, $N \subseteq N^{*}$, and for each $\varepsilon_{2}>0$ there exists $f \in N$ such that (3.5) has exactly $f$ nontrivial $2 \pi$-pertodic solutions lytng in $P\left(\varepsilon_{2}\right)$.

(111) for any $\bar{\varepsilon} \in\left(0, \varepsilon_{1}\right)$ there extsts a neighborhood $N_{\bar{\varepsilon}}$ of $f_{0}, N_{\varepsilon} \subseteq N^{*}$ such that if $\mathrm{F} \in \mathrm{N}_{\bar{E}}$ and if $\gamma$ is a $2 \pi$-pertodic solution of (3.5) 1ying In $P\left(\varepsilon_{1}\right)$ then $\gamma$ lies in $P(\bar{\varepsilon})$.

The solutions of (3.5) are the representation in polar coordinates of the orbits of the solutions of (3.2). It is not clear apriori that (a') Imp11es (a) because these properties Involve neighborhoods of the origin In $(r, v)$ space and of the origin in $(x, y, \zeta)$ space respectively while the substitution (3.4) Is singular at $r=0$. Nonetheless, we can prove the following proposition.

Proposition 2. Property (a') implies (a).

Proof. Without loss of generality we may assume $\varepsilon_{1}<1$. Define the mapping $\psi:(x, y, \zeta) \rightarrow(r, v),(x, y) \neq(0,0)$, given by the substitution $(3.4)$, that ts $r=\sqrt{x^{2}+y^{2}}$ and $v=\zeta / \sqrt{x^{2}+y^{2}}$. Then $\psi^{-1}\left(P\left(E_{1}\right)\right) \subset B^{n}\left(\varepsilon_{1}\right)$ since $r^{2}+\|v\|^{2}<\varepsilon_{1}^{2}$ implies $r^{2}+\|\zeta\|^{2}=r^{2}+r^{2}\|v\|^{2} \leq r^{2}+\|v\|^{2}<\varepsilon_{1}{ }^{2}$. Each $2 \pi$-pertodic solution of (3.5) 1ying in $P\left(E_{1}\right)$ corresponds in polar coordinates 
to the orbit of a periodic solution of (3.2) lying in $\mathrm{B}^{\mathrm{n}}\left(\varepsilon_{1}\right)$ whose perlod is included in some interval $\mathrm{I}_{\delta_{1}}$. Let $E, \delta, L$, and $\mathrm{N}_{0}$ be the constants and netghborhood of $f_{0}$ defined in Proposttion and asaume $\varepsilon_{1}<E$. In view of (3.6) and the observation $\theta\left(\theta, 0,0, \mathrm{f}_{0}\right) \equiv 1$ we may choose $\delta_{1}$ and $N^{*}$ such that $\delta_{1}<\delta$ and $N^{*} C N_{0}$. Let $a_{1}=\varepsilon_{1} / \sqrt{1+L^{2}}$. If $\mathrm{f} \subseteq N *$ and any perfodic orbit of (3.2) 1ying in $b^{n}\left(a_{1}\right)$ whose perlod is in $I_{s}$ then by Proposition $1 \psi(\gamma) \subset P\left(\varepsilon_{1}\right)$. Indeed, if $(x, y, \zeta) \subset \gamma$ and $r=\sqrt[1]{x^{2}+y^{2}}$, then $(r, v)=\psi(x, y, \zeta)$ antisfieg $\|v\|^{2}+r^{2}=\frac{\|\zeta\|^{2}}{r^{2}}+r^{2}<L^{2} r^{2}+r^{2}=r^{2}$ $\left(1+L^{2}\right)<a_{1}^{2}\left(1+L^{2}\right)<\varepsilon_{1}^{2}$. Thus, propety $(a)(f)$ follows from $\left(a^{\prime}\right)(1)$.

Proof of (a)(11). Plck any $\overline{a_{2}}<a_{2}<\varepsilon_{1}, \sqrt{1+L^{2}}$ and $\tilde{N} c^{*}$ such that for every $f \in \tilde{N}$ we have: (1) the periodic orblts of (3.2) $1 y \operatorname{lng}$ In $\mathrm{B}^{\mathrm{n}}\left(\overline{\mathrm{a}}_{2}\right)$, which correspond to the 21 -pertudtc orbtts of $(3.5)$ lying in $\mathrm{p}\left(\bar{a}_{2}\right)$ have period in $I_{\delta_{2}}$. This follows by using $(3.6)$ and the fact that $\theta\left(\theta, 0,0, f_{0}\right) \equiv 1$. (2) $E \in N$ implies that all the $2 \pi$-pertodic orbits of (3.5) 1ying in $P\left(c_{1}\right)$ lie in $P\left(a_{2}\right)$. Thts can be done in view of ( $\left.a^{\prime}\right)(11 i)$.

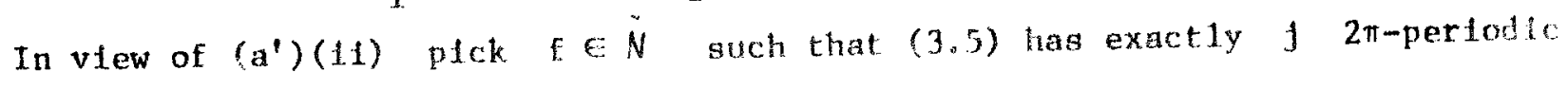
orbits lying in $\mathrm{P}\left(\overrightarrow{\mathrm{a}}_{2}\right)$. If $\gamma$ is any perlodic otbit of (3.2) 1ying in $B^{n}\left(a_{2}\right)$ whose pertod to in $I_{\delta_{2}}$ then $\psi(\gamma) \subseteq P\left(c_{1}\right)$. In ylew of $(2)$ $\psi(\gamma) \subseteq \mathrm{P}\left(\overrightarrow{\mathrm{a}}_{2}\right)$. This completes the proof of $(a)(11)$.

Proof of (a) (111). Assume $\bar{a}_{1}<\bar{a}<E_{1} / \sqrt{1+L^{2}}$ and $\bar{N} \subset N^{*}$ such that conditions (1) and (2) above hold with $\tilde{N}$ replaced by $\bar{N}$ and $\delta_{2}, \bar{a}_{2}$, and $a_{2}$ replaced by $\bar{\delta}, \bar{a}_{1}$, and $\bar{a}$ respectively, Then, if $Y$ is a perfodic orbit of (3.2) Iying in $B^{n}\left(a_{1}\right)$ with perfod in $I_{\delta_{1}}$, then $\psi(\gamma) \subseteq \mathrm{p}\left(\varepsilon_{1}\right)$ which fmplieg $\psi(\gamma) \subseteq \mathrm{p}\left(\bar{a}_{1}\right)$. Then, in view of $(1)$ and $(2)$, 
$\because\left[\theta^{n}\left(\bar{a}_{1}\right) \subseteq B^{n}(\bar{\varepsilon})\right.$ with perlod tn $T_{0}$. Thit completes the proof of

\section{toposition 2 .}

A solucton $(r(\theta), v(0))$ of $(3,5)$ that extstes on $10,2 \pi]$ vitil be

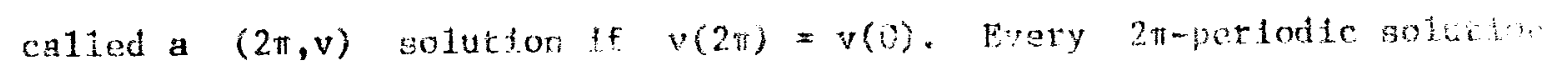

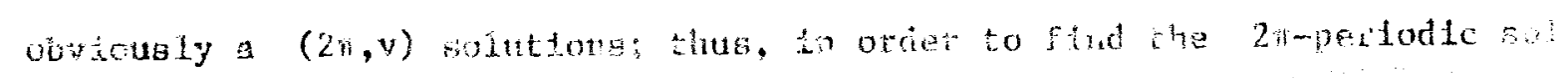

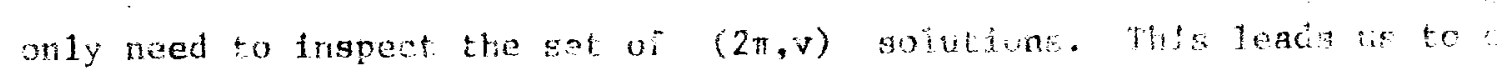
the following propostatio.

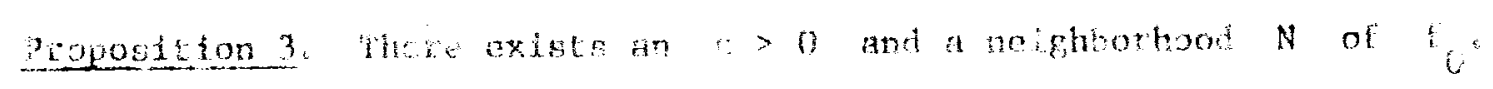

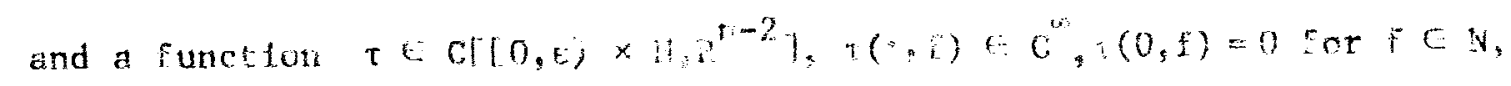

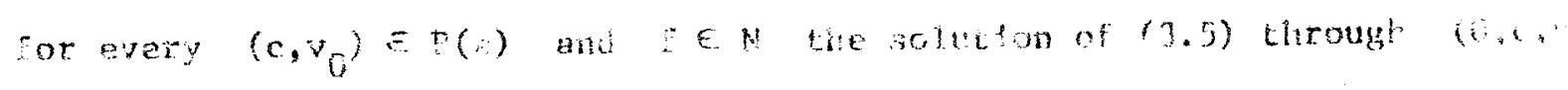
1 a $(2 \pi, v)$ solution 1 and anty $j$ i $v_{n}=t(0, f)$.

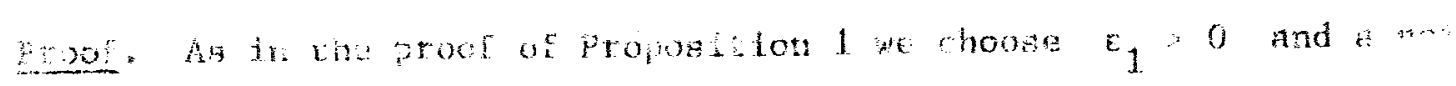

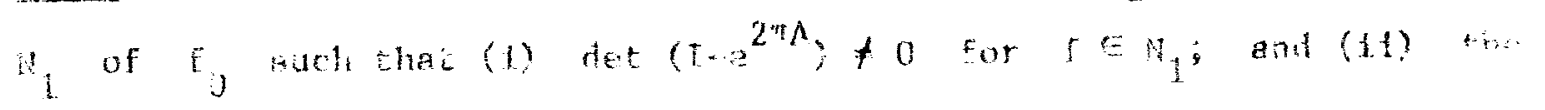

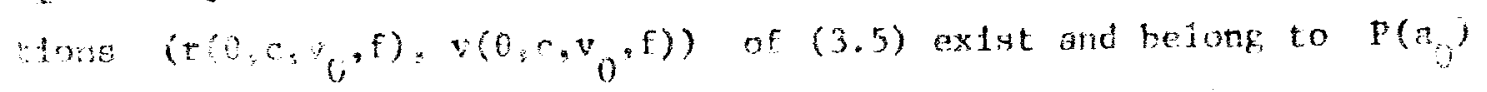

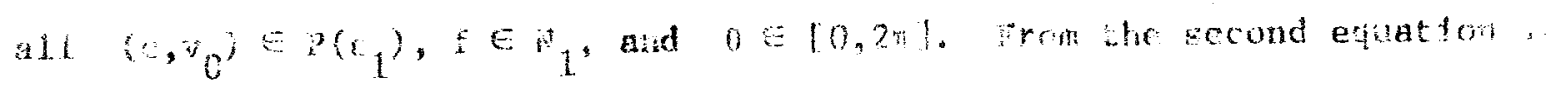

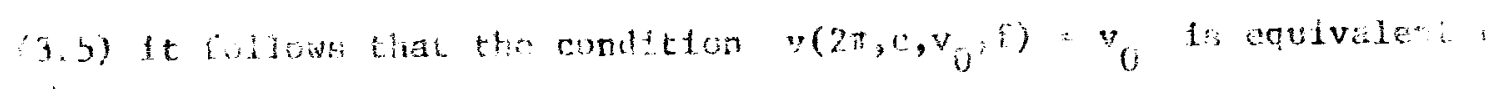
requtrentic that $F\left(c_{,}, f\right)=0$, where

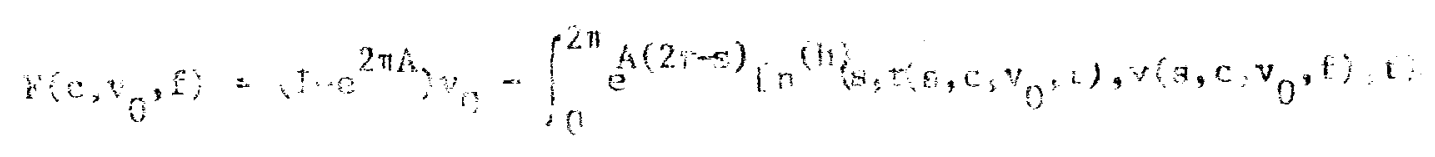

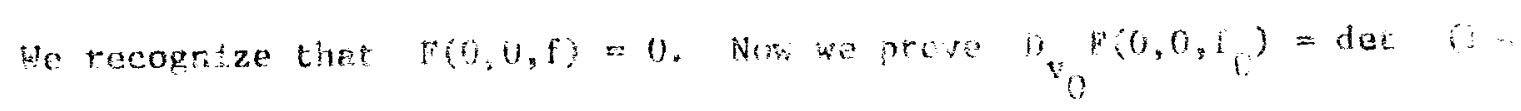

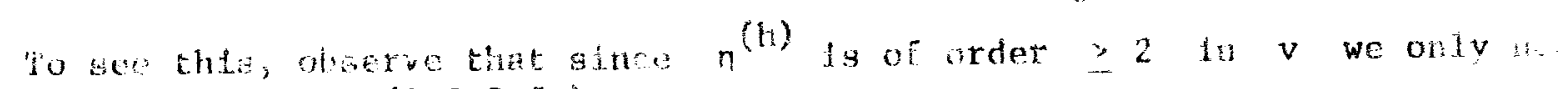

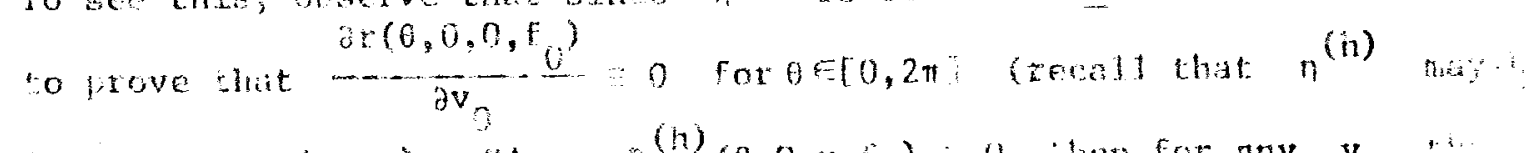

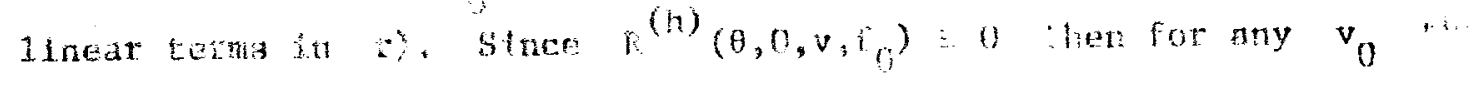

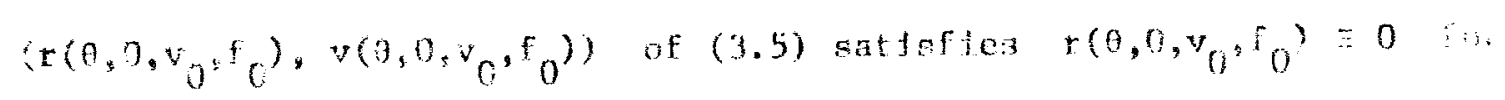

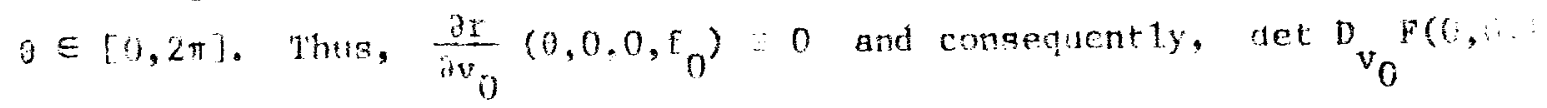


$\operatorname{det}\left(t-e^{2 \pi A_{0}}\right) \neq 0$. Therefore, the conclusion of Proposttion 3 follows from the Implicit function theorem and the fact that $F(0,0, f)=0$.

Denote by $(r(\theta, c, f), v(\theta, c, f))$ the $(2 \pi, v)$ solution of (3.5) passing through $(0, c, t(c, f))$. Because of Proposition 3 we can write

$$
\begin{aligned}
& r(\theta, c, f)=u_{1}(\theta, f) c+\ldots+u_{h}(\theta, f) c^{h}+o\left(c^{h}\right) \\
& v(\theta, c, f)=v_{1}(\theta, f) c+\ldots+v_{h-1}(\theta, f) c^{h-1}+o\left(c^{h-1}\right),
\end{aligned}
$$

where $u_{1}(0, f)=1, u_{1}(0, f)=0$ for $1>1$ and

$$
v_{1}(0, f)=v_{1}(2 \pi, f) \quad \text { for } \quad 1 \geq 1
$$

Conslder now the displacement function relative to the $(2 \pi, v)$ solutions which is defined in a right interval of $c=0$ and in a neighborhood of $f_{0}$

$$
v(c, f)=r(2 \pi, c, f)-c .
$$

Then the $2 \pi$-periodic solutions of (3.5) correspond to the zeros of $V(c, f)$. We now prove the following result.

Proposition 4. Assume the origin of $\left(\mathrm{S}_{h}\right)$ Is etther h-asymptotically stable or h-completely unstable. Then $h$ is odd and

$$
\frac{\partial^{1} v}{\partial c^{1}}\left(0, f_{0}\right)=0,1=1 \ldots h-1 \text { and } \frac{\partial^{h} v}{\partial c}\left(0, f_{0}\right) \neq 0 \text {. }
$$

proof. Substitute (3.7) into the second equation in (3.5) for $f=f_{0}$ and equate coeffictents of $c$. Since $n^{(h)}\left(\theta, r, 0, f_{0}\right)$ ts of order $>h-1$ in $r$ we $f$ ind that $\nu_{1}\left(\theta, f_{0}\right) \equiv 0$ for $1=1, \ldots h-1$. Indeed,

$$
\frac{\partial v_{1}}{\partial \theta}\left(\theta, f_{0}\right)=A_{0} v_{1}
$$

Implying $v_{1}\left(\theta, f_{0}\right)=v_{1}\left(0, f_{0}\right) e^{A 0^{\theta}}$. 
Condition (3.8) and the fact that $\operatorname{det}\left(I-e^{2 \pi A_{0}}\right) \neq 0$ Imp11es that $v_{1}\left(0, f_{0}\right)=i$ and consequent $1 y, \nu_{1}\left(\theta, f_{0}\right) \equiv 0$. Since $\nu_{1}\left(\theta, f_{0}\right) \equiv 0$ we then have

$$
\frac{\partial \nu_{2}}{\partial \theta}\left(\theta, f_{0}\right)=A_{0} \nu_{2}
$$

and as before, $\nu_{2}\left(\theta, f_{0}\right) \equiv 0$. Continuing in this manner we obta1n $\nu_{1}\left(\theta, f_{0}\right) \equiv 0_{;}$ $1=1 \ldots h-1$. Thus, in order to compute the functions $u_{1}\left(\theta, f_{0}\right)$ we may put $v=0$ into the first equation in (3.5) for $f=f_{0}$. We then obtain the equation

$$
\frac{d r}{d \theta}=R^{(h)}\left(\theta, r, 0, f_{0}\right),
$$

which to precisely the equation in polar coordinates of the orbits of $\left(s_{h}\right)$. Since $x=y=0$ is efther h-asymptotically atable or h-completely unstable for $\left(s_{h}\right)$ we have that $h$ is odd and

$$
u_{1}\left(\theta, f_{0}\right) \equiv 1, u_{1}\left(2 \pi, f_{0}\right)=0,1=2 \ldots h-1, u_{h}\left(2 \pi, f_{0}\right) \neq 0,
$$

(see [9] for more detal1s), thus implying (3.10) holds.

Final1y, we have the following result concerning the roots of $v(c, f)$

for $f$ close to $\mathrm{f}_{0}$.

Proposition 5. Asoume the orfgin of $\left(s_{n}\right)$ is efther h-asymptotica11y atable of h-completely unstable. Then there exlst $\vec{c}>0$ and a nelghborhood $\bar{N}$ of $f_{0}$ such that: (1) $V(c, f)$ is defined for any $c \in \mid 0, \bar{c}]$ and $f \in \bar{N}$; (2) for every $c_{1} \in(0, \bar{c})$ there exlats a nefghborhood $N_{c_{1}} \subseteq \bar{N}$ such that for $f \in N_{c_{1}} a 1$. roots of $V(c, f)$ lying in $[0, \bar{c}]$ 11e in $\left[0, c_{1}\right)$.

The proof of Proposition 5 utilizes Proposition 4 in order to show that tir $\bar{c}>0$ suffictently small and $c \in[0, \bar{c}]$ we have $\left|V\left(c, f_{0}\right)\right| \geq \mu c^{h}$ for an ap proprlate constant $\mu>0$. The 
continuity of $V(c, f)$ in $c$ and $f=f_{0}$ allows us to conclude that for every $c_{1} \in[0, \bar{c}]$ there exists $N_{c_{1}} \subset N$ such that for $f \in \mathbb{N}_{c_{1}},|V(c, f)| \geq \operatorname{lr}^{\prime} \mu c_{1}^{h}$ for $c \in\left[c_{1}, \bar{c}\right]$. We leave the detafls to the reader.

\section{PROOF OF THEOREM 1}

If In the transformation (3.4) we assume $r<0$ Instead of $r>0$ we obtain a new displacement function $\tilde{v}(c, f)$ defined for $c<0$. We will extend the domain of $V$ by setting $V(c, f)=\tilde{V}(c, f)$ for $c<0$. It is easy to recognize that this extended function $1 \mathrm{~s}$ continuous and for fixed $f$ is $C^{\infty}$ in c. In addition, we observe that for any $2 \pi$-pertodtc solution of (3.5) there exist $c_{1}>0, c_{2}<0$ such that $V\left(c_{1}, f\right)=V\left(c_{2}, f\right)=0$.

Assume Case I. We sha11 prove property (a') holds and in view of Propositin 3 the proof of the first part of Theorem 1 will be complete.

proof of property (a) (1). Since the origin is a solution of (3.5) for any $f$, an application of Rolle'g Theorem, In view of (3.10), Implles that there extsts an $E_{1}>0$ and a nelghborhood $N *$ of $F_{0}$ such that for any $f \in N^{*}$, $V(c, f)$ has at most $h-1$ nonzero roots counting multiplicity in $\left[-E_{1}, \varepsilon_{1}\right]$. For each positive root of $V(c, f)$ there is a negative root of $V(c, f)$ corresponding to the same periodic orbit. Thus, there are at nost $k=\frac{h-1}{2}$ nontrivial $2 \pi$-perfodtc solutions of $(3.5)$ lying $\ln P\left(c_{1}\right)$ for $f \in N^{*}$. Thus, property $\left(a^{*}\right)(1)$ holds.

Proof of property $\left(a^{\prime}\right)(11)$. We essentially adapt to our problem a proceedure used in [1]. Suppose the origin of $S_{h}$ is h-asymptotically stable (the case In which the origin of $\left(S_{h}\right)$ is h-completely unstable has a similar proof). 
Consider a perturbed system of (3.1) of the form

$$
\begin{aligned}
& \dot{x}=-y+x_{0}^{(h)}(x, y, \zeta)+\sum_{i=1}^{f} a_{1} x\left(x^{2}+y^{2}\right)^{k-1} \\
& \dot{y}=x+Y_{0}^{(h)}(x, y, \zeta)+\sum_{i=1}^{j} a_{1} y\left(x^{2}+y^{2}\right)^{k-1} \\
& \dot{\zeta}=A_{0} \zeta+W_{0}^{(h)}(x, y, \zeta),
\end{aligned}
$$

where $k=\frac{h-1}{2}, f$ is any integer, $1 \leq f<k$, and $a_{1}$ are constants to be determined (the case $f=0$ follows by letting $f=f_{0}$ ). We will denote by $v\left(c, a_{1}, \ldots, a_{f}\right)$ the displacement function relative to the $(2 \pi, v)$ solutions of (3.5) which correspond to (4.1). We will denote by $s\left(a_{1}, \ldots, a_{f}\right)$ the first two equations in $(4.1)$ for $\zeta=0$. Since the origin is h-asymptotically stable for $s(0, \ldots, 0)$ then from Proposition 4

$$
v(c, 0,0, \ldots, 0)=g_{0} c^{h}+o\left(c^{h}\right), g_{0}<0
$$

Thus, for $c_{0}>0$ and suffictently small we have $v\left(c_{0}, 0, \ldots, 0\right)<0$. There exists an $n_{1}>0$ such that $v\left(c_{0}, a_{1}, \ldots, a_{f}\right)<0$ for $\left|a_{1}\right|<n_{1}$, $1=1, \ldots, j$. Fix now $a_{1}, 0<a_{1}<n_{1}$. Then

$$
v\left(c, a_{1}, 0, \ldots, 0\right)=g_{1} c^{h-2}+o\left(c^{h-2}\right)
$$

where $g_{1}=2 \pi a_{1}>0$. This can be recognized by replacing the expreasion for $r$ in (3.7) Into $S(a, 0, \ldots, 0)$ and taking finto account that $u_{1}(2 \pi, f)=0$ $1=1, \ldots, h-3$ (see $[9]$ for more detalls). There extats $c_{1}, 0<c_{1}<c_{0}$ such that $V\left(c_{1}, a_{1}, 0, \ldots, 0\right)>0$ and thus, we can find $\eta_{2}>0, n_{2}<n_{1}$, such that for $\left|a_{1}\right|<n_{2}, 1=2, \ldots, 1, v\left(c_{1}, a_{1}, a_{2}, \ldots, a_{f}\right)>0$. Fix now $a_{2}<0$, $\left|a_{2}\right|<n_{2}$ Then 


$$
v\left(c, a_{1}, a_{2}, 0, \ldots, 0\right)=g_{2} c^{h-3}+o\left(c^{h-3}\right)
$$

where $g_{2}=2 \pi a_{2}<0$. Then there exists $c_{2}, 0<c_{2}<c_{1}$ such that $v\left(c_{2}, a_{1}, a_{2}, 0, \ldots, 0\right)<0$ and thus, we can find $n_{3}>0, n_{3}<n_{2}$ such that for $\left|a_{1}\right|<n_{3}, 1=3, \ldots, f, v\left(c_{2}, a_{1}, \ldots, a_{3}\right)<0$. Continuing this procese we can $f$ ind a set of numbers $\bar{c}_{1}, \bar{c}_{2}, \ldots, \bar{c}_{1}, c_{1}<\bar{c}_{1}<c_{1-1} 1=1, \ldots, j$ (and thus $\left.0<\bar{c}_{1+1}<\bar{c}_{1} 1=1, \ldots, j-1\right)$ such that $v\left(\bar{c}_{1}, a_{1}, \ldots, a_{j}\right)=0$, $1=1, \ldots, f$. Since $c=0$ ls a root of $v\left(c, a_{1}, \ldots, a_{j}\right)$ of order $h-2 j$ (recal1 $\left.u_{1}(2 \pi, f)=0 \quad 1=1, \ldots, h-21-1\right)$ and for each positive root of $v\left(c, a_{1}, \ldots, a_{f}\right)$ we have a negative root then we lmmed lately have that the $\bar{c}_{1} 1=1, \ldots, f$ are the only posttive roots of $v\left(c, a_{1}, \ldots, a_{j}\right)$. Moreover, we can obtain that the $\bar{c}_{1}$ can be made close to $c=0$ by picking $c_{0}$ sufficlently sma11. This completes the proof of $\left(a^{\prime}\right)(11)$.

The proof of $\left(a^{\prime}\right)(111) \quad i s$ an immediate consequence of Propostition 5.

Thus, property ( $\left.a^{\prime}\right)$ holda and so the firgt part of Theorem 1 is proved.

We now show case 11 followg from case $I$. For any posttive integer $f$ we assume in (3.1) $h=2 t+1$ and congtier perturbed syatems of the form

$$
\begin{aligned}
& \dot{x}=-y+x_{0}^{(h)}(x, y, c)+b x\left(x^{2}+y^{2}\right)^{\frac{h-1}{2}} \\
& \dot{y}=x+y_{0}^{(h)}(x, y, c)+b y\left(x^{2}+y^{2}\right)^{\frac{h-1}{2}} \\
& \dot{t}=A_{0} r+w_{0}^{(h)}(x, y, c),
\end{aligned}
$$

where $b$ is a constant. We then have that fot the corresponding reduced syatem $\left(\mathrm{S}_{\mathrm{h}}\right)$ the orfgtn $1 \mathrm{~s}$ elther h-asymptotically atable or h-completely unstable if $b<0$ or $b>0$ respectively. Thus, wr have reduced the problem to Case I. 
Since $f$ and $b$ are arbitrary, property (A) holds, thus concluding the proof of Theorem 1 .

5. PROOF OF COROLLARIES.

The proof of Corollary 1 follows from (2.7) and Theorem 1 by observing that there $1 \mathrm{~s}$ an equivalence between the h-asymptotic stability (h-complete Instability) of the origin of (3.1) on $H_{h}$ and the h-asymptotic stability (h-complete instablitty) of the ortgin of $\left(s_{h}\right)$.

\section{Proof of Corollary 2.}

We observe that the origin of the system (3.1) Is h-asymptotically atable In the whole if and only if the origin of $\left(S_{h}\right)$ is h-asymptoticaliy atable. Indeed, If the orlgin of $\left(s_{h}\right)$ is h-asymptotically stable, there extsts a constant $G<0$ and a polynomial

$$
F(x, y, b)=x^{2}+y^{2}+F_{3}(x, y, \zeta)+\ldots F_{h}(s, y, \zeta)
$$

$\left(F_{1}\right.$ is a homogeneous polynomial of degree 1) such that the dertvative of $F$ along the solutions of $(3.1)$ can be written as

$$
\dot{H}(x, y, z)=G\left(x^{2}+y^{2}\right)^{\frac{h+1}{2}}+o\left(x^{2}+y^{2}+\|\|^{2}\right)^{\frac{h+1}{2}}
$$

(note that $\dot{F}(x, y, 0)$ dlffers from the derivative of $F$ along the solutions of $\left(s_{h}\right)$ by terms of order greater than $\left.h+2\right)$. Moreover, there exists a quadratic form in $r, Q(\zeta)$, such that along the solutions of system (3.1)

$$
\dot{q}(x, y, r)=-\|r\|^{2}+x_{1}(x, y, r)+x_{2}(x, y, r)
$$


where $x_{1}$ is of order $\geq 3$ and of order $\geq 2$ In $\zeta ;$ and $x_{2}$ 1s of order $>h+1$ (see Lyapunov [7] for a detalled analysts of the above statementa). Setting $n=F+Q$ we have that along the solutions of aystem (3.1), for $x, y, \zeta \quad$ sma11,

$$
\begin{aligned}
\dot{n}(x, y, \zeta) & =-\| \eta^{2}+G\left(x^{2}+y^{2}\right)^{\frac{h+1}{2}}+x_{1}(x, y, \zeta)+\sigma(x, y, \zeta) \\
& =\frac{-\|\zeta\|^{2}}{2}+G\left(x^{2}+y^{2}\right)^{\frac{h+1}{2}}+\sigma(x, y, r),
\end{aligned}
$$

where $\sigma$ is of order $>h+1$ and includes $x_{2}(x, y, \zeta)$. Then $n$ is positive definfte and its derivative along golutions of (3.1) is negative definite. This property holds if we perturb (2.1) with terms of order greater than $h$. On the other hand, this property will not hold by appropriately choosing perturbations of order $\leq \mathrm{h}$. Thus, the orfgtn of (2.1) is h-asymptotically stable.

Using gimllar argumenta we can show that if the origtn is h-asymptotically stable in the whole then the orfgin $1 \mathrm{~s}$ h-asymptotically gtable on $\mathrm{H}_{\mathrm{h}}$. Analogoualy, we can prove the or 1 gln 13 h-unstable in the whole if and only if the ortgln of $\left(S_{h}\right)$ is h-completely unstable. Tn view of corollary 1 this completes the proof of Corollary 2. 
BTBLIOGRAPHY

[1] Andronov, A., Leontovtch, E., Gordon, I., and Maier, A., Theory of blfurcations of dynamical systems in the plane, larael program of. Sclentffic Translations, Jerusalem, (1971).

[2] Bernfeld, S. and Salvadorl, L., Generalized llopf blfurcation and hasymptotic stabli1ty, J. Nonlinear Analysis, T.M.A. 4(1980), 1091-1107.

[3] Bernfeld, S., Negrini, P. \& Salvadort, L., Stabllfty and generalized Hopf bifurcation through a reduction princtple, Proc. of the Conf. "Nonlinear Differential Equations: Invarlance, Stablitty, and B1furcation" Trento, May 25-30, 1980, Academic Press (to appear).

[4] Blbikov, Y., Local theory of nonlinear analytic ordinary differential equations, Lecture Notes in Mathematics 702, Springer-Verlag, New York (1979).

[5] Chafee, N. , Generalized Hopf bffurcation and perturbation in a ful1 nelghborhood of a given vector field, Indlana Untr. Math. J. 27(1978), 173-194.

[6] Hale, J. K., Toples in dynamic blfurcation theory, NSF-CBMS Conference, Arlington, Texas, June 16-20, 1980.

171 Lyapunov, A. M., Probleme general de la stab111té du mouvement, Ann. of Math Studies, 17, Princeton Untv. Presa, Princeton, New Jersey, (1947).

[8] Marsden, G. and McCracken, M., The Hopf bifurcation and 1ts app1icatIons, Notes In App11ed Math. Sc1. 19, Springer Verlag, New York, 1976.

[9] Negrind, P. and Salvador1, L., Attractivity and Hopf blfurcation, J. Non1fnear Ana1. T.M.A. 3 (1979), 87-100.

[10] Sansone, G. and Cont1, R., Non11near different1a1 equations, MacM111an, New York, (1964). 